\section{Notes on the genera Asterolibertia and Cirsosia (Fungi: Ascomycota)}

\section{V.B. Hosagoudar}

Tropical Botanic Garden and Research Institute, Palode Thiruvananthapuram, Kerala 695562, India Email:vbhosagoudar@rediffmail.com

The ascomycetous genera having orbicular thyriothecium with central stellate dehiscence at the centre are classified under the family Asterinaceae, while, the genera having oval to elongated thyriothecium dehisce all along at the centre are classified under the family Lembosiaceae. Both these families are grouped under the order Asterinales. These fungi are characterized by having brown superficial hyphae, possessing appressoria or nutritive hyphae. Fruiting body is of thyriothecium, scutate with radiating cells on the upper surface.

Some of the genera possess lateral appressoria while a few have in the hyphal cells (intercalary). Asterinaceous fungi having intercalary appressoria belong to two genera: Asterolibertia (Asterinaceae) and Cirsosia (Lembosiaceae); the former genus having orbicular thyriothecia dehiscing stellately at the centre and the latter genus having oval or elongated thyriothecia dehiscing longitudinally at the centre (Arx \& Muller 1975; Hosagoudar et al. 2001). Stevens \& Ryan (1939) have provided a check list of the genera and species but enough changes have been taken place in the study of this group of fungi since there no monographic work of this group. Hosagoudar \& Abraham (2000) have listed the number of taxa belonging to the genus Asterina, Song et al. (2003) have listed the till then known species of the

Date of publication (online): 26 August 2010 Date of publication (print): 26 August 2010 ISSN $0974-7907$ (online) | 0974-7893 (print)

Editor: Richard Mibey

Manuscript details:

Ms \# 02465

Received 24 May 2010

Finally accepted 06 July 2010

Citation: Hosagoudar, V.B (2010). Notes on the genera Asterolibertia and Cirsosia (Fungi: Ascomycota). Journal of Threatened Taxa 2(9): 11531157

Copyright: (C) V.B. Hosagoudar 2010. Creative Commons Attribution 3.0 Unported License. JoTT allows unrestricted use of this article in any medium for non-profit purposes, reproduction and distribution by providing adequate credit to the authors and the source of publication.

Acknowledgements: I thank the Director, Tropical Botanic Garden and Research Institute, Palode for the facilities.

\section{OPEN ACGESS I FREE DOWNLOAD (C) (i) (4)}

genus Lembosia and Goos (1999)

for Echidnodella. The present account is also in the same line to help in the identification of the fungi belonging to these two genera.

The Genus Asterolibertia (Fig. 1).

Asterolibertia Arn., Les Asterinees, 1: 161, 1918; Hansf., Mycol. Pap. 15: 189, 1946; Muller \& Arx, Beitr. Krypt. Schw. 11: 97, 1962; Luttrell in Ainsworth et al. (eds.). The Fungi. An advanced Treatise 4: 207, 1973; Arx \& Muller, Stud. Mycol. 9: 43, 1975; Bilgrami, Jamaluddin \& Rizwi, Fungi of India p. 54, 1991; Hosag., Abraham \& C.K. Biju, J. Mycopathol. Res. 39: 61, 2001; Singh, Duke, Bhandari \& Jain, J. Econ. Taxon. Bot. 30: 185, 2008.

Steyaertia Bat. \& Maia, Univ. Recife, Inst. Mycol. Publ. 295: 5, 1960

Wardina Arn., Les Asterinees 1:165, 1918.

Leaf parasites. Mycelium ectophytic, appressoria intercalary, setae absent. Thyriothecia orbicular with radiating cells, astomatous, dehisce stellately at the center; asci globose, octosporous, bitunicate; ascospores conglobate, uniseptate, brown.

Type sp.: A. couepiae (Henn.) Arn.

\section{Enumeration of the species \\ Host: Anacardiaceae}

Asterolibertia hydnocarpi Hosag. \& Abraham, J. Mycopathol. Res. 35: 55, 1997.

On leaves of Hydnocarpus macrocarpus, H. pentandra, India.
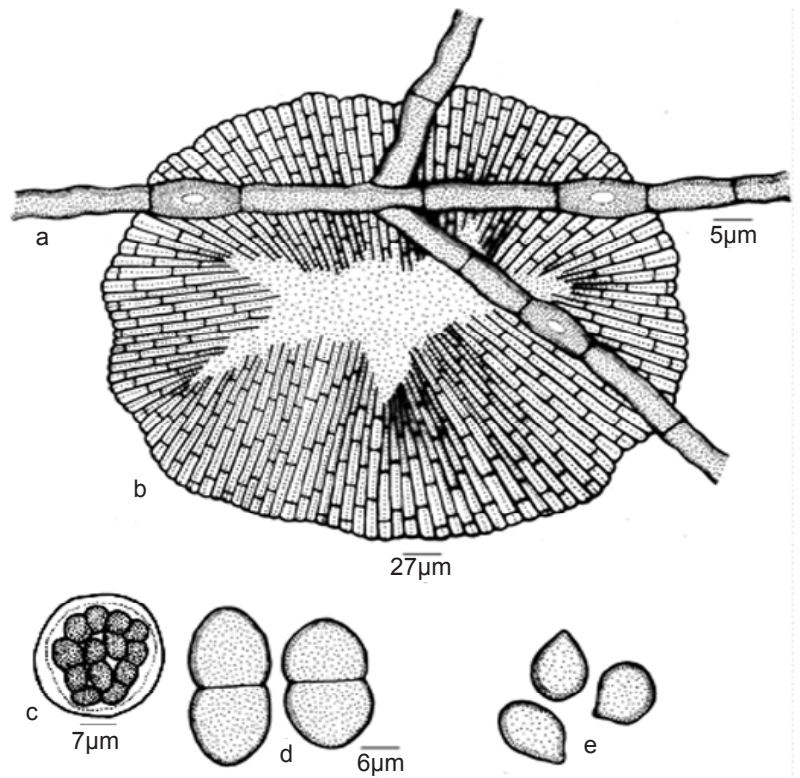

Figure 1. The genus Asterolibertia Arn.

a - Intercalary appressoriate mycelium; b - Thyriothecium;

c - Ascus; d - Ascospores; e - Pycnothyriospores 
Asterolibertia mangiferae Hansf. \& Thirum., Farlowia 3: 303, 1948.

On leaves of Mangifera indica, India

Asterolibertia nothopegiae Hosag. \& Abraham, New Botanist 24: 109, 1997.

On leaves of Nothopegia aureo-fulva, India.

\section{Host: Annonaceae}

Asterolibertia nodulosa (Cooke) Hansf., Proc. Linn. Soc. London 160: 140, 1948.

Asterina nodulosa Speg., Fungi Puigg. No. 353 in

Biol. Acad. Sci. Cordoba 11: 563, 1889.

On leaves of Guatteriae dolichopoda, Costa Rica.

\section{Host: Bignoniaceae}

Asterolibertia peruviana Hansf., Sydowia 9: 81, 1955.

On leaves of Bignoniaceae member, Peru.

\section{Host: Burseraceae}

Asterolibertia santiriae (Sydow) Hansf., Reinwardtia

3: 126, 1954.

Asterinella santiriae Sydow, Ann. Mycol. 15: 248, 1917.

Prillieuxina santiriae (Sydow) Ryan in Stev. \& Ryan, Illinois Biol. Monographs 17: 80, 1913.

On leaves of Santiria nitida, Philippines.

\section{Host: Chrysobalanaceae}

Asterolibertia licaniae (Cooke) Hansf., Proc. Linn.

Soc., London 160: 140, 1949.

Asterina licaniae Cooke, Grevillea 12: 851884.

On leaves of Licania sp., Brazil.

Asterolibertia licaniicola Hansf., Proc. Linn. Soc

London 160: 140, 1949.

On leaves of Licania sp., Brazil.

Asterolibertia schroeteri (Rehm) Arx in muller \& Arx,

Beitr. Krypt. der Schweiz, p.98, 1962.

Seynesia schroeteri Rehm, Hedwigia 37: 326, 1898

Asterina schroeteri (Rehm) Theiss., Abh. Zool. Bot.

Ges. Wien. 7: 3, 1913

On leaves of Chrysobalanus sp., South America.

\section{Host: Dipterocarpaceae}

Asterolibertia anisopterae (Sydow) Hansf., Proc.

Linn. Soc., London 160: 139, 1949.

Morenoella anisopterae Sydow, Ann. Mycol. 12: 560, 1914.

Asterina anisopterae Sydow, Ann. Mycol. 12: 556, 1914.

On leaves of Anisoptera thursifera, Philippines.

Asterolibertia flabellariae (Sydow) Hansf., Proc. Linn. Soc., London 158: 44, 19497.

Lembosia flabellariae Sydow, Ann. Mycol. 36: 189, 1938.

On leaves of Flabellaria paniculata, Sierra Leone
Asterolibertia vateriae Hosag. in Hosag., H. Biju \& Appaiah, J. Mycopathol. Res. 44: 13, 2006.

On leaves of Vateria indica, India.

\section{Host: Fabaceae}

Asterolibertia spatholobi Hansf., Reinwardtia, 3: 127, 1954.

On leaves of Spatholobus ferrugineus, Java.

\section{Host: Lauraceae}

Asterolibertia cryptocaryae (Cooke) Hansf., Proc. Linn. Soc. N.S.W. 79: 106, 1954.

Asterins cryptocaryae Cooke in Herb., Kew.

Asterinella cryptocaryae (Cooke) Theiss., Broteria 10: 107, 1912.

Prillieuxina cryptocaryae (Cooke) Ryan in Stev \& Ryan, Illinois Biol. Monographs 17: 79, 1939.

On leaves of Cryptocaryae sp., Australia

Asterolibertia cryptocaryae (Cooke) Hansf. var nodulifera Hansf., Proc. Linn. Soc. N.S.W. 79: 106, 1954.

On leaves of Cryptocarya rigida, Australia.

\section{Host: Malphigiaceae}

Asterolibertia ulei Hansf., Proc. Linn. Soc. London 16: 142, 1949.

On leaves of Malphigiaceae member, Brazil.

Asteroliberta couepiae (Henn.) Arn., Les Asterinees 1: $165,1918$.

Asterina couepia Henn., Hedwigia, 34: 104, 1895.

Steyaertia couepiae Bat. \& Maia, Univ. Recife, Inst. Micol. Publ. 295: 5, 1960.

On leaves of Couepia ovalifolia, C. rufa, Brazil.

\section{Host: Myrtaceae}

Asterolibertia crustacea (Ell. \& Ev.) Hansf., Sydowia 9: 80, 1955.

Asterella crustacea Ell. \& Ev., Publ. Field. Mus., Columbia Bot. 2: 15, 1900.

Asterina crustacea (Ell. \& Ev.) Sacc. \& Trott., Syll. Fung. 22: 539, 1913.

On leaves of Psidium guajava, San Domingo.

\section{Host: Poaceae}

Asterolibertia sporoboli Castellani \& Graniti in Graniti, Nuovo G. Bot. Ital. 57: 50, 1950.

On leaves of Sporobolus ruspolanus, British Somaliland.

\section{Host: Polygalaceae}

Asterolibertia bredemeyerae (Rehm) Arx in Muller \& Arx, Beitr. Krypt. der Schweiz, p.98, 1962.

Asterina bredemeyerae Rehm, Hedwigia 40: 161, 1901.

Asterinella bredemeyerae Orejuela, Mycologia 36: 443, 1944. 
On leaves of Bredemeyera spp., South America

\section{Host: Rosaceae}

Aserolibertia parinarii (Sydow) Hansf., Proc. Linn. Soc. London 159: 36, 1947.

Asterina parinari Sydow, Ann. Mycol. 36: 180, 1938.

On leaves of Parinarium excelsum, Sierra Leone, Africa.

\section{Host: Rubiaceae}

Asterolibertia burchelliae (Doidge) Doidge, Bothalia 4: 315, 1942.

Asterinella burchelliae Doidge, Trans. Royal Soc. South Africa 8: 267, 1920.

Prillieuxina burchelliae (Doidge) Ryan in Stev. \& Ryan, Illinois Biol. Monograph 17: 77, 1939.

On leaves of Burchellia capensis, Terenna pavettoides, Rubiaceae.

Asterolibertia gibbosa (Gaill.) Hansf., Proc. Linn. Soc. London 160: 141, 1948.

Asterina gibbosa Gaill., Bull. Soc. Mycol. France 13: 180, 1897.

On leaves of Bananacantha spinosa, Randia sp., Brazil.

Asterolibertia megathyria Doidge, Bothalia 4: 314, 1942.

Asterina gibbosa Gaill. var. megathyria Doidge, Trans.

Royal. Soc. South Africa 8: 248, 1920.

Asterinella ugandensis Hansf., Proc. Linn. Soc.

London 153: 48, 1941

On leaves of Tricalysia spp., South Africa.

Asterolibertia randiae (Doidge) Arx in Muller and Arx, Beitr. Krypt. der Schweiz. p.99, 1962.

Asterolibertia megathyria Doidge var. randiae Doidge,

Bothalia 4: 315, 1942.

On leaves of Randia dumetorum, Canthium spp., South Africa

\section{Host: Styracaceae}

Asterolibertia hiiranensis Yamamoto, Sci. Rep. Hyogo. Univ. Agric., Agric. Biol. Ser. 3: 29, 1957.

Asterina hiiranensis Yamamoto., Sci. Rep. Hyogo.

Univ. Agric., Agric. Biol. Ser. 2: 35, 1956.

On leaves of Styrax hayataiana, Formosa, Taiwan.

\section{Doubtful species}

Asterolibertia inaemelis (Mont.) Arn., Ann.Sci.Nat.

Bot.Ser.Sev.10, 7: 721, 1925.

This is known on the members of three host families (Muller \& Arx, 1962).

Asterolibertia myocoproides (Sacc.\& Bercl.) Arx in Muller and Arx, Beitr. Krypt. der. Schweiz, p.100, 1962.

This is known on the members of two host families (Muller \& Arx, 1962). 1957.

Asterolibertia thaxteri Hansf., Sydowia 11: 63
Known on unknown host.

\section{The genus Cirsosia (Fig. 2)}

Cirsosia Arn., Ann. Ecol. Nat. Agric. Montpellier 16: 127,1918; Hansf., Mycol. Pap. 15: 189, 1946; Muller \& Arx, Beitr. Krypt. der. Schweiz. 11:113, 1962; Luttrell in Ainsworth et al. (eds.). The Fungi. An advanced Treatise 4: 207, 1973; Arx \& Muller, Stud. Mycol. 9: 44, 1975; Hosag., Abraham \& C.K. Biju, J. Mycopathol. Res. 39: 62, 2001; Singh, Duke, Bhandari \& Jain, J. Econ. Taxon. Bot. 30: 186, 2008.

Cirsosiella Arn., Les Asterinees 1:127, 1918.

Halbania Arn., Les Asterinees 1: 163, 1918.

Leaf parasites. Mycelium ectophytic, appressoria intercalary. Thyriothecia oval, ellipsoidal, $X$ or $Y$ shaped, elongated with radiating cells, astomatous, dehisce longitudinally at the center; asci oval, octosporous, bitunicate; ascospores brown, conglobate, uniseptate.

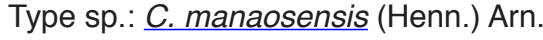

\section{Enumeration of the species \\ Host: Arecaceae}

Cirsosia arecacearum Hosag. \& Pillai, Mycol. Res. 98:128, 1994.

On leaves of Calamus thwaitesii, India

Cirsosia globuliferae (Pat.) Arn. in Muller \& Arx, Beitr. Krypt. der Schweiz, p.114. 1962.

Lembosia globuliferae Pat., J. Bot. p.65, 1890.

Asterina globuliferae (Pat.) Theiss., Abh. Zool. Bot. Ges. Wein 7: 56, 1913.

Cirsosiella globuliferae (Pat.) Arn., Les Asterinees 1:

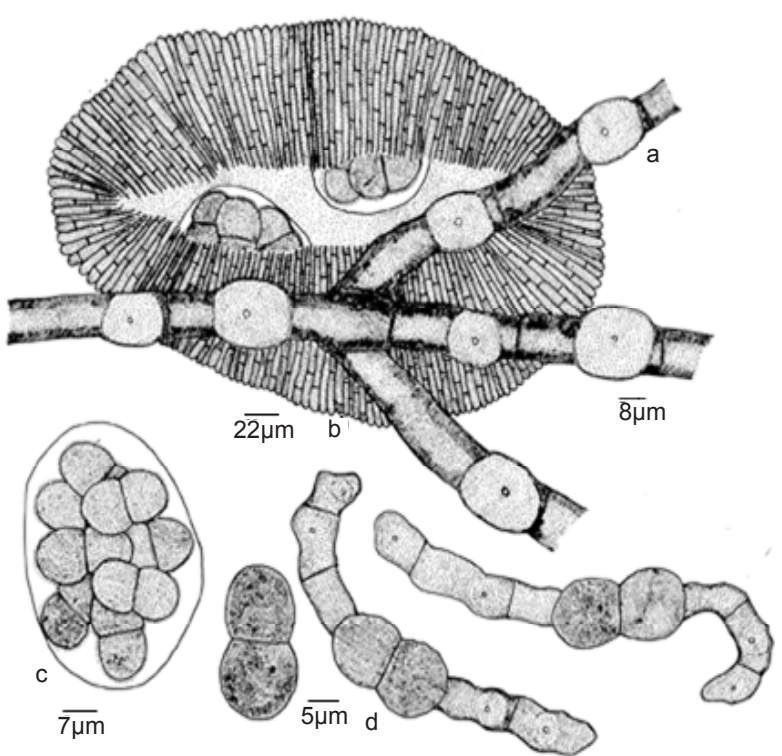

Figure 2 .The genus Cirsosia Arn.

a - Intercalary appressoriate mycelium; b - Thyriothecium; c - Ascus; d. Ascospores 
Table 1. Host and species index

\begin{tabular}{|c|c|}
\hline Anisoptera thursifera & $\begin{array}{l}\text { Asterolibertia anisopterae } \\
\text { Morenoella anisopterae } \\
\text { Asterina anisopterae }\end{array}$ \\
\hline Bananacantha spinosa & $\begin{array}{l}\text { Asterolibertia gibbosa } \\
\text { Asterina gibbosa }\end{array}$ \\
\hline Bignoniaceae member & Asterolibertia peruviana \\
\hline Bredemeyera spp. & $\begin{array}{l}\text { Asterolibertia bredemeyerae } \\
\text { Asterina bredemeyerae } \\
\text { Asterinella bredemeyerae }\end{array}$ \\
\hline Burchellia capensis & $\begin{array}{l}\text { Asterolibertia burchelliae } \\
\text { Asterinella burchelliae } \\
\text { Prillieuxina burchelliae }\end{array}$ \\
\hline Calamus sp. & $\begin{array}{l}\text { Cirsosia globuliferae } \\
\text { Lembosia globuliferae } \\
\text { Asterina globuliferae } \\
\text { Cirsosiella globuliferae } \\
\text { Asterina bakeri } \\
\text { Asterolibertia bakeri } \\
\text { Cirsosia transversalis } \\
\text { Asterina transversalis } \\
\text { Morenoella transversalis } \\
\text { Cirsosiella transversalis }\end{array}$ \\
\hline Calamus thwaitesii & Cirsosia arecacearum \\
\hline Canthium spp. & $\begin{array}{l}\text { Asterolibertia randiae } \\
\text { Asterolibertia megathyria Doidge var. } \\
\text { randiae }\end{array}$ \\
\hline Chrysobalanus sp. & $\begin{array}{l}\text { Asterolibertia schroeteri } \\
\text { Seynesia schroeteri } \\
\text { Asterina schroeteri }\end{array}$ \\
\hline Clonodia verrucosa & $\begin{array}{l}\text { Cirsosia manaosensis } \\
\text { Lembosia manaosensis }\end{array}$ \\
\hline Couepia ovalifolia & $\begin{array}{l}\text { Asterolibertia couepiae } \\
\text { Asterina couepiae } \\
\text { Steyaertia couepiae }\end{array}$ \\
\hline Couepia rufa & $\begin{array}{l}\text { Asteroliberta couepiae } \\
\text { Asterina couepia } \\
\text { Steyaertia couepiae }\end{array}$ \\
\hline Cryptocarya rigida & $\begin{array}{l}\text { Asterolibertia cryptocaryae (Cooke) } \\
\text { Hansf. var nodulifera }\end{array}$ \\
\hline Cryptocarya sp. & $\begin{array}{l}\text { Asterolibertia cryptocaryae } \\
\text { Asterina cryptocaryae } \\
\text { Asterinella cryptocaryae } \\
\text { Prillieuxina cryptocaryae }\end{array}$ \\
\hline Flabellaria paniculata & $\begin{array}{l}\text { Asterolibertia flabellariae } \\
\text { Lembosia flabellariae }\end{array}$ \\
\hline Guatteriae dolichopoda & $\begin{array}{l}\text { Asterolibertia nodulosa } \\
\text { Asterina nodulosa }\end{array}$ \\
\hline Hydnocarpus macrocarpus & Asterolibertia hydnocarpi \\
\hline Hydnocarpus pentandra & Asterolibertia hydnocarpi \\
\hline Licania sp. & $\begin{array}{l}\text { Asterolibertia licaniae } \\
\text { Asterina licaniae } \\
\text { Asterolibertia licaniicola }\end{array}$ \\
\hline Malphigiaceae member & Asterolibertia ulei \\
\hline Mangifera indica & Asterolibertia mangiferae \\
\hline Nothopegia aureo-fulva & Asterolibertia nothopegiae \\
\hline Parinarium excelsum & $\begin{array}{l}\text { Aserolibertia parinarii } \\
\text { Asterina parinari }\end{array}$ \\
\hline Psidium guajava & $\begin{array}{l}\text { Asterolibertia crustacea } \\
\text { Asterella crustacea } \\
\text { Asterina crustacea }\end{array}$ \\
\hline
\end{tabular}

\begin{tabular}{|l|l|}
\hline Randia dumetorum & $\begin{array}{l}\text { Asterolibertia randiae } \\
\text { Asterolibertia megathyria Doidge var. } \\
\text { randiae }\end{array}$ \\
\hline Randia sp. & $\begin{array}{l}\text { Asterolibertia gibbosa } \\
\text { Asterina gibbosa }\end{array}$ \\
\hline Santiria nitida & $\begin{array}{l}\text { Asterolibertia santiriae } \\
\text { Asterinella santiriae } \\
\text { Prillieuxina santiriae }\end{array}$ \\
\hline Spatholobus ferrugineus & Asterolibertia spatholobii \\
\hline Sporobolus ruspolanus & Asterolibertia sporoboli \\
\hline Styrax hayataiana & $\begin{array}{l}\text { Asterolibertia hiiranensis } \\
\text { Asterina hiranensis }\end{array}$ \\
\hline Terenna pavettoides & $\begin{array}{l}\text { Asterolibertia burchelliae } \\
\text { Asterinella burchelliae } \\
\text { Prillieuxina burchelliae }\end{array}$ \\
\hline Tricalysia spp. & $\begin{array}{l}\text { Asterolibertia megathyria } \\
\text { Asterina gibbosa } \\
\text { Asterinella ugandensis }\end{array}$ \\
\hline Unknown hosts & $\begin{array}{l}\text { Asterolibertia inaemelis } \\
\text { Asterolibertia myocoproides } \\
\text { Asterolibertia thaxteri }\end{array}$ \\
\hline Vateria indica & $\begin{array}{l}\text { Asterolibertia vateriae } \\
\text { Asterina irregularis } \\
\text { Morenoella irregularis } \\
\text { Cirsosiella irregularis } \\
\text { Halbanina irregularis }\end{array}$ \\
\hline
\end{tabular}

128, 1916.

Asterina bakeri Sydow, Ann. Mycol. 14: 367, 1916.

Asterolibertia bakeri (Sydow) Hansf., Proc. Linn. Soc. London 160: 139, 1948.

On leaves of Calamus sp., Asia.

Cirsosia transversalis (Sydow) Bat. \& Maia, Rev. Biol. 2: 128, 1960.

Asterina transversalis Sydow, Leafl. Philippine Bot. 62: 1542, 1911.

Morenoella transversalis (Sydow) Theiss Ann. Mycol. 11: 457, 1913.

Cirsosiella transversalis (Sydow) Arn., Les Asterinees 1: 128, 1918.

On leaves of Calamus sp., Philippines.

\section{Host: Dipterocarpaceae}

Cirsosia irregularis (Sydow) Arx in Muller \& Arx, Beitr. Krypt. der Schweiz 115, 1962.

Asterina irregularis Sydow, Leafl. Philipp. Bot. 62: 1540, 1911.

Morenoella irregularis (Sydow) Theiss., Ann. Mycol.11: 458, 1913.

Cirsosiella irregularis (Sydow) Arn., Les Asterinees 1: 128, 1918.

Halbanina irregularis (Sydow) Hansf., Reinwardtia 3: 119, 1954.

On leaves of Vatica obtusifolia, Philippines 


\section{Host: Malphigiaceae}

Cirsosia manaosensis (Henn.) Arn., Les. Asterinees 1: $27,1918$.

Lembosia manaosensis Henn., Hedwigia 43: 265, 1904.

On leaves of Clonodia verrucosa, Brazil.

\section{REFERENCES}

Arx, J.A.V. \& E. Muller (1975). A Re-evaluation of the Bitunicate Ascomycetes with key to the Families and Genera. Stud. Mycol. 9: 1-159.

Hosagoudar, V.B. \& T.K. Abraham (2000). A list of Asterina Lev. species based on the literature. Journal of Economic and Taxonomic Botany 24: 557-587.

Hosagoudar, V.B., T.K. Abraham \& C.K. Biju (2001). Reevaluation of the family Asterinaceae. Journal of Mycopathol. Research 39: 61-63.

Song, B. \& V.B. Hosagoudar (2003). A list of Lembosia species based on the literature. Guizhou Science 21: 93-101.

Goos, R.D. (1999). Notes on the genus Echidnodella (Asterinaceae). Mycotaxon 73: 455-454.

Stevens, F.L. \& M.H. Ryan (1939). The Microthyriaceae. Illinois Biological Monograph 17: 1-138. 